\title{
Ovarian Squamous Cell Carcinoma Associated with Endometriosis: Poor Response to Chemotherapy
}

\section{Seob Jeon}

Department of Obstetrics and Gynecology, Soonchunhyang University Cheonan Hospital, Soonchunhyang University College of Medicine, Cheonan, Korea

\begin{abstract}
The occurrence of ovarian squamous cell carcinoma associated with ovarian endometriosis is extremely rare. Several studies revealed poor prognosis for ovarian squamous cell carcinoma especially when associated with endometriosis. We report a case of ovarian squamous cell carcinoma that invades the parametrium that is also associated with ovarian endometriosis. A 41-year-old woman with stage IIB ovarian squamous cell carcinoma was treated with debulking surgery followed by six courses combination chemotherapy composed of paclitaxel and carboplatin. Twenty months after the last course of chemotherapy (3 months after normal positron emission tomography-computed tomography [CT] scanning), upper abdominal pain developed and abdomen CT scan revealed multiple metastasis in liver and paraaortic lymph node. Although two cycles of paclitaxel and cisplatin was administrated, she died of disease three months after the recurrence.
\end{abstract}

Keywords: Squamous cell carcinoma; Endometriosis; Chemotherapy

\section{INTRODUCTION}

Most ovarian squamous cell carcinomas (SCC) originate from cystic teratoma. However only $2 \%$ of cystic teratoma become malignant. SCC can also originate from endometriosis or Brenner tumors, and it rarely occurs as a primary pure form [1]. Malignant changes of endometriosis in the ovary or rectovaginal septum may ocurr. The resulting tumor is either endometrioid adenocarcinoma or clear cell carcinoma [2]. The prognosis of ovarian SCC is worse than that of other epithelial ovarian malignancies. The prognosis of endometriosis-related cases is even worse than cystic teratoma-related cases [3]. Currently reported treatments are radiotherapy or cytotoxic chemotherapy after surgery. However, it is hard to find consistent treatment and their efficacies are questionable. Recently, some authors have reported good responses to combination chemotherapy in treatment of recurrent and metastatic ovarian SCC.

We report of primary ovarian SCC associated with endometrio- sis showing poor response to combination paclitaxel and cisplatin chemotherapy at the time of recurrence.

\section{CASE REPORT}

\section{Clinical findings}

A 41-year-old (gravida 0, para 0) woman was admitted to the department of obstetrics and gynecology of Soonchunhyang University Cheonan Hospital with the chief complaint of lower abdominal pain for 20 days. Two years ago, she underwent surgery for uterine synechia. She had no familial history of cancer. Bimanual pelvic examination demonstrated a 4-month pregnant sized mass with mild tenderness inside of the pelvic cavity and normal cervix and vagina. Complete blood cell count and blood chemistry were within normal limits. Three tumor markers were a little higher than normal: carcer antigen (CA 125), 95.19 IU/mL; CA 19-9, 40.36 $\mathrm{IU} / \mathrm{mL}$; SCC, $2.6 \mathrm{ng} / \mathrm{mL}$. Other tumor markers (carcinoembrionic antigen, alpha fetoprotein, and beta-human chorionic gonadotro- 

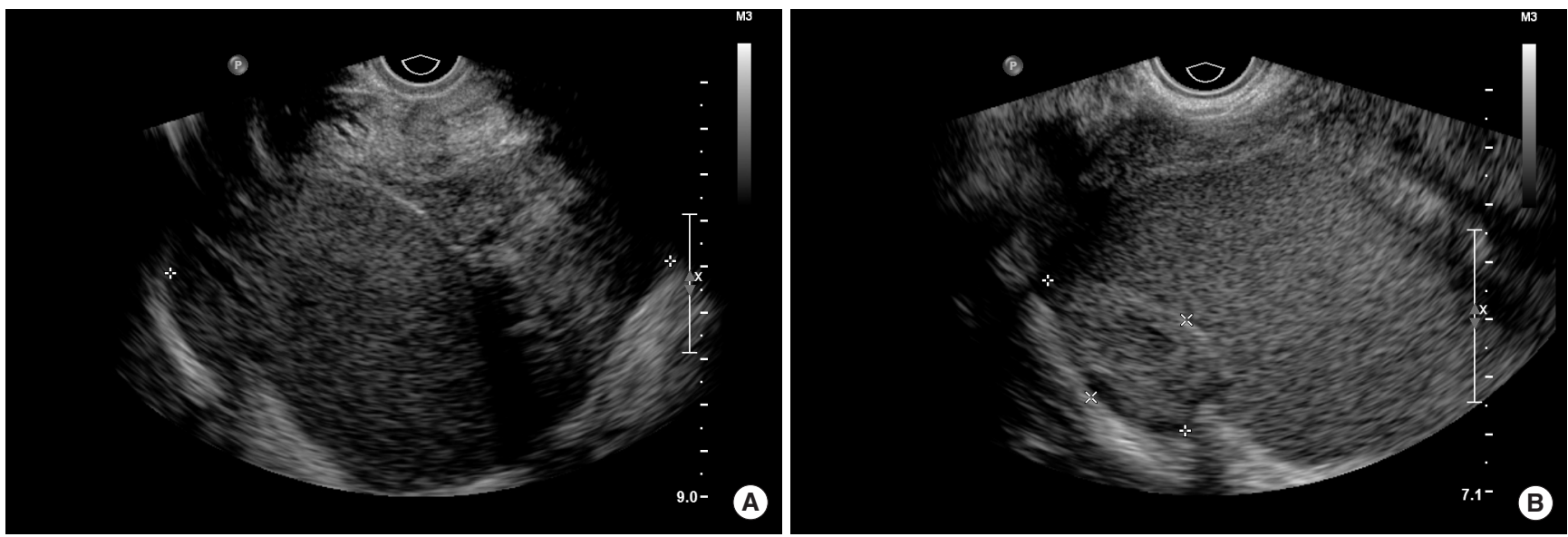

Fig. 1. Transvaginal ultrasound shows (A) heterogenous echoic solid mass (over $10 \mathrm{~cm}$ ) in left pelvic cavity and (B) mass of same character in right pelvic cavity.

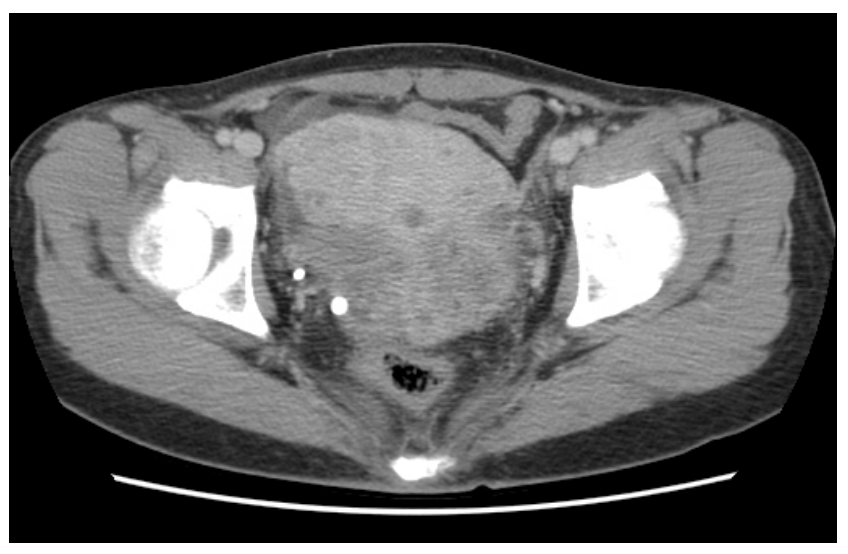

Fig. 2. Pelvic computed tomography scan shows $10 \times 9-\mathrm{cm}$ sized irregular marginated solid and cystic mass involving the left distal ureter was seen in pelvic cavity.

phin) were in normal range. Cervical smear demonstrated no evidence of dysplasia. A $10 \times 9-\mathrm{cm}$ solid mass with heterogeneous shadow was noted at the left side of pelvic cavity by transvaginal ultrasonography (Fig. 1). Computed tomography (CT) showed a $10-\mathrm{cm}$ irregular marginated solid and cystic complex pelvic mass and hydronephrosis of the left kidney. Ascites and abnormal pelvic and paraaortic lymphadenopathy were not observed (Fig. 2). Intravenous pyelogram revealed left hydronephrosis with distal left ureteral compression by pelvic mass. Gastrofiberscopic and sigmoidoscopic examination showed no abnormal finding.

The patient underwent a laparotomy one week after admission due to the possibility of a malignant pelvic tumor. A solidified mass, about $10 \mathrm{~cm}$ in diameter, originated from the left ovary, and attached to the left pelvic peritoneum, was observed in the pelvic cavity, but there was no capsular rupture and no ascites. We start- ed adhesiolysis for removal of the mass after a retroperitoneum incision inside of the pelvis, but the tumor had already spread out to the left parametrium, which resulted in left distal ureteral obstruction. Therefore, we detached the left ureter up to the entrance of the bladder and removed the tumor en bloc. The tumor was diagnosed as a SCC of the left ovary from the frozen section. We performed a total hysterectomy, bilateral salpingoophorectomy, multiple peritoneal biopsy, infracolic omentectomy, and pelvic lymphadenectomy after peritoneal washing cytology.

\section{Pathological findings}

The dimensions of the removed mass from left ovary were $9.0 \times$ $5.0 \times 5.0 \mathrm{~cm}^{3}$. Also the cross-sectional view showed that it was partially solid and partially cystic. The solid part was $7.5 \times 4.5 \times 4.0$ $\mathrm{cm}^{3}$, the color was light yellow, and traces of bleeding and necrosis were observed. The cystic part contained turbid bloody liquid. The right ovary was $2.5 \times 2.0 \times 1.8 \mathrm{~cm}^{3}$, and looked normal. The surface of the fallopian tube on both sides had severe adhesions and the lumen of the right side fallopian tube appeared hydrosalpinx. The resected uterus was $7.0 \times 6.0 \times 3.0 \mathrm{~cm}^{3}$. Two solid masses were observed at the myometrium. The size of two lumps was 4.0 $\times 3.0 \times 2.5 \mathrm{~cm}^{3}$ and $0.8 \times 0.4 \times 0.4 \mathrm{~cm}^{3}$, respectively, and the myometrium was thickened partially with random pattern. The cervix seemed to be in normal condition.

The microscopy results indicated that the mass from the left ovary was moderately differentiated SCC and that there were partial traces of bleeding and necrosis. The most cystic part of tumor was covered with macrophage containing hemosiderin and partial endometrial glandular cell. As a result, a diagnosis of endometriosis 


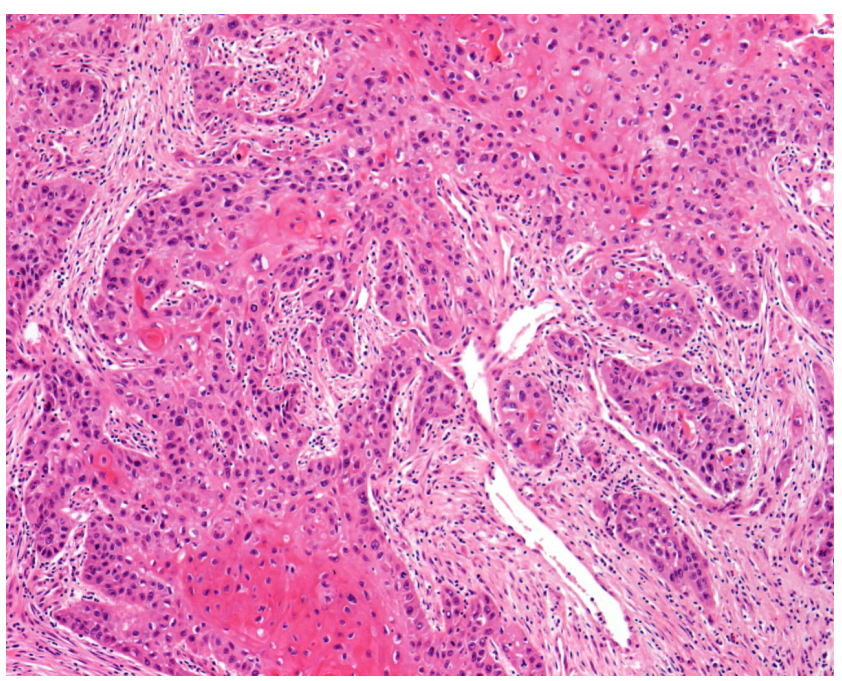

Fig. 3. The tumor is composed of atypical squamous cell nests with cytoplasmic keratinization $(H \& E, \times 100)$.

was made. Diffused traces of endometriosis also were observed at both the fallopian tubes and the tissue around them (Fig. 3). There were two uterine myomas and adenomyosis in fundus. There were no other abnormal conditions, including dysplasia at the uterine cervix. SCC was found in the left parametrium and the tissue around the left ureter. No other tumor metastasis was observed at the greater omentum, lymph node, or biopsied peritoneum.

Four weeks after surgery, postoperative combination chemotherapy using paclitaxel $\left(175 \mathrm{mg} / \mathrm{m}^{2}\right)$ and carboplatin (area under the curve of 5) was conducted every three weeks for a total of six treatments. The numeric value of CA 125, CA 19-9, and SCC became normal after the first course of chemotherapy. There had been no recurrence for 20 months after the surgery, but the patient was admitted via the emergency room at the 20th month because of acute exacerbation of upper abdominal pain. CT scan revealed multiple metastases in the liver and upper abdominal lymph nodes (Fig. 4). The estimated tumor marker, CA 125 was 121.9 IU/ $\mathrm{mL}$, CA 19-9 was 2,928.0 U/mL, and SCC was $0.5 \mathrm{ng} / \mathrm{mL}$. We transferred patients to another hospital at patient's request. The patients had two cycles of paclitaxel-cisplatin at the transferred hospital, but did not respond to chemotherapy. The patient passed away two months later without any additional treatment.

\section{DISCUSSION}

World Health Organization's ovarian cancer classification contains SCC as a new type of ovarian surface epithelial stromal tu-

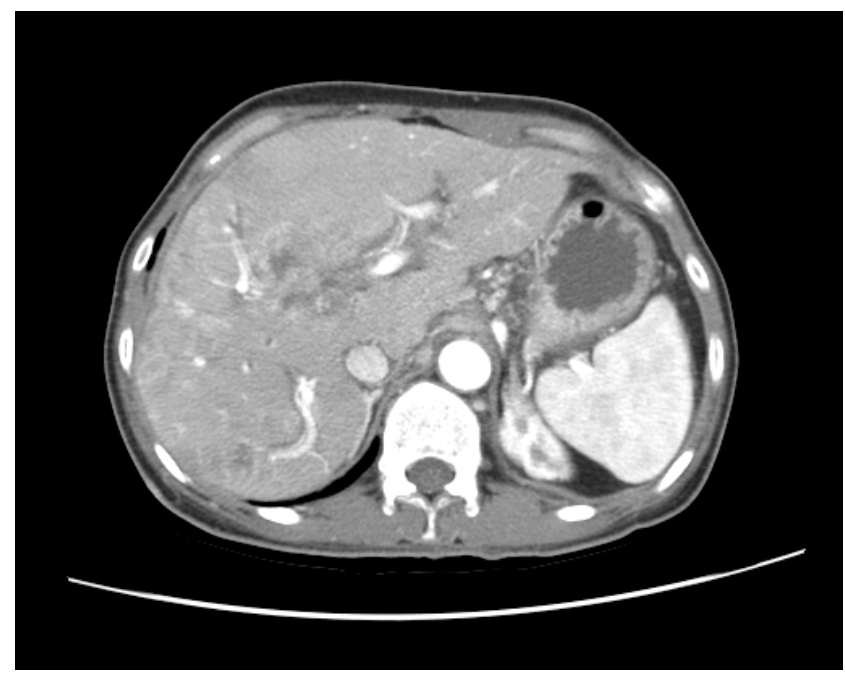

Fig. 4. Computed tomography image revealed multiple metastases in the liver.

mor. Ovarian SCC is common in their 50's. The stage of disease and the degree of differenciation may affect to the survival outcome [4].

Most malignant tumors that originate from endometriosis are endometrioid adenocarcinoma and clear cell carcinoma, and they rarely develop to SCC. Sampson [5] and Scott [6] presented some conditions for verifying whether the SCC originated as endometriosis or not: (1) endometriosis and squamous epithelial cell have to be developed from the same ovary, and the locational correlation between the tissue from benign endometriosis and the tissue from malignant SCC has to have same pattern as a malignant tumor that develops from the endometrium; (2) the malignant tumor has to have grown inside the tissue of endometriosis and it should not to be transferred from the outside of endometriosis to the inside; (3) there has to be tissue present that alludes to endometriosis like endometrial stroma, findings of old hemorrhage and hemosiderin pigmentation, and so on; finally (4) the part changing to benign epithelium has to be observed by microscopy. However, these criteria are outdated and it is very hard to apply them because SCC originating from endometriosis is extremely rare.

Our case satisfies (1) and (3) of Sampson's and Scott's criteria but cannot identify or does not satisfy (2) and (4). However, the case is highly indicative of endometriosis.

To our knowledge there are no more than 20 cases of ovarian SCCs associated with endometriosis in the literature. Acien et al. [7] reviewed 16 cases of SCCs arising from or associated with endometriosis. Ten cases met Sampson's and Scott's criteria. In six of 
the cases, endometriosis was closely apposed to the tumor. Most of patients diagnosed with SCC associated with endometriosis were younger than 50 years old and had advanced disease. Their stages were classified as follows: three cases were stage I, six cases were stage II, three cases were stage III, and four cases were stage IV. The most common symptom was abdominal pain and some patients presented flu like symptom. Spreading pattern and clinical finding of these kind of malignancy were frequent invasion surrounding tissue such as parametrium and ureter, like our case, in addition to absence of ascites and poor response of radiotherapy and chemotherapy [7]. In terms of survival, some authors reported poor survival outcome in patients with ovarian SCC compared with other epithelial malignancies $[3,8]$. Some researchers reported favorable survival outcome for patients with malignancies in early stages [810], but Kashimura et al. [1] reported poor 5-year-survival rate even in patients with early stage diagnoses. Although no long-term survival has been reported for patients with advanced stage ovarian SCC originating from endometriosis and only 3 patients out of 16 patients with SCC originated from endometriosis were alive when the case was published, and mean survival of patients died of their disease was 4.8 months, a case report of Eltabbakh et al. [11] shows the disease free case of hepatic metastasis from ovarian SCC associated with endometriosis for 2 years after complete remission by 12 cycles of paclitaxel and cisplatin combination chemotherapy following optimal debulking surgery (except for the hepatic metastasis). Their decision to use paclitaxel and cisplatin was based on hepatic metastatic location and excellent results from ovarian carcinoma and SCC of the head and neck.

Pins et al. [3] reported 37 cases of ovarian SCCs. Nineteen were associated with benign cystic teratoma, seven were associated with endometriosis, and eleven were pure type. Pins et al. [3] also analyzed the survial rate of SCCs (benign cystic teratoma relating type, endometriosis relating type, and pure type). Although the number of cases was too small and the period of observation was too short, Pins et al. [3] reported that the SCCs associated with endometriosis had the worst survival rate of all. Survival was also related to the stage of disease and grade of tumor.

The reported treatments for ovarian SCCs were chemotherapy, radiotherapy, or both following surgery. However, the effectiveness of adjuvant radiotherapy and chemotherapy has not been established. There is not any efficient preparation of chemotherapy following surgery for ovarian SCCs until now, but chemotherapy based on platinum is recommended by many studies.
In the systemic review of ovarian SCC originating from mature cystic teratoma, postoperative radiotherapy was not beneficial. In fact, it might adversely affect survival [10].

Jeon et al. [12] also reported survived case of hepatic metastasis from pure ovarian SCC for 10 months without recurrence after eight cycles of combiatinon chemotherapy with paclitaxel and cisplatin after surgery. Ohtani et al. [13] reported the patient with rapidly growing ovarian SCC associated endometriosis treated with combination chemotherapy of cisplatin, bleomycin, mitomycin $\mathrm{C}$, and vincristion achieved complete remission with the combination chemotherapy of weekly paclitaxel and carboplatin. However, Todo et al. [14] reported the failure of this combination chemotherapy. He conducted surgery for stage IIIc ovarian SCC without residual tumor and tried same combination chemotherapy continuously, but the patient died during the fifth chemotherapy treatment because of the metastasis to both lungs [14].

In our case, left broad ligament and the tissue around the left ureter were involved, but there was no additional metastasis to other organs in abdominal cavity, greater omentum, or pelvic lymph nodes. Therefore it was stage IIb. We conducted adjuvant chemotherapy consisting of paclitaxel and carboplatin based on review of above reports. Although positron emission tomographyCT scan was normal on patient's last visit, three months later patient presented hepatic and multiple paraaortic lymph node metastases. These finding reflects rapid progression of ovarian SCC originating from endometriosis and the need for a new therapeutic strategy. Radiation therapy is extremely important because SCC is a radiosensitive tumor and also because it controls local recurrence. There is one opinion insisting on concurrent chemoradiation therapy as an additional treatment after the surgery for ovarian SCC. Hence, concurrent chemoradiation might be reasonable option as an adjuvant treatment of the disease [15].

Ovarain SCC is extremely rare, but endometriosis related cases are even rarer and postoperative treatement should involve combination of paclitaxel and platinum agent and because of the radiosensitivity of this tumor, we may consider concurrent chemoradiation after surgery as well.

\section{REFERENCES}

1. Kashimura M, Shinohara M, Hirakawa T, Kamura T, Matsukuma K. Clinicopathologic study of squamous cell carcinoma of the ovary. Gynecol Oncol 1989;34:75-9.

2. Brooks JJ, Wheeler JE. Malignancy arising in extragonadal endometrio- 
sis: a case report and summary of the world literature. Cancer 1977;40: 3065-73.

3. Pins MR, Young RH, Daly WJ, Scully RE. Primary squamous cell carcinoma of the ovary: report of 37 cases. Am J Surg Pathol 1996;20:823-33.

4. Hoskins WJ, Perez CA, Young RC, Barakat RR, Markman M. Principles and practice of gynecologic oncology. 4th ed. Baltimore (MD): Lippincott Williams \& Wilkins; 2005.

5. Sampson JA. Endometrial carcinoma of the ovary, arising in endometrial tissue in that organ. Arch Surg 1925;10:1-72.

6. Scott RB. Malignant changes in endometriosis. Obstet Gynecol 1953;2: 283-9.

7. Acien P, Abad M, Mayol MJ, Garcia S, Garde J. Primary squamous cell carcinoma of the ovary associated with endometriosis. Int J Gynaecol Obstet 2010;108:16-20.

8. Kikkawa F, Ishikawa H, Tamakoshi K, Nawa A, Suganuma N, Tomoda Y. Squamous cell carcinoma arising from mature cystic teratoma of the ovary: a clinicopathologic analysis. Obstet Gynecol 1997;89:1017-22.

9. Pettersson F. Annual report on the results of treatment in gynecological cancer. Stockholm: International Federation of Gynecology and Obstetrics; 1991.

10. Hackethal A, Brueggmann D, Bohlmann MK, Franke FE, Tinneberg HR, Munstedt K. Squamous-cell carcinoma in mature cystic teratoma of the ovary: systematic review and analysis of published data. Lancet Oncol 2008;9:1173-80.

11. Eltabbakh GH, Hempling RE, Recio FO, O'Neill CP. Remarkable response of primary squamous cell carcinoma of the ovary to paclitaxel and cisplatin. Obstet Gynecol 1998;91(5 Pt 2):844-6.

12. Jeon JD, Choi EG, Jo EM, Kim YT, Kim JW. A case of squamous cell carcinoma of the ovary showing complete remission to combination chemotherapy composed of paclitaxel and cisplatin. Korean J Obstet Gynecol 2000;43:1492-6.

13. Ohtani K, Sakamoto H, Masaoka N, Shimada K, Kanaeda T, Kurihara M, et al. A case of rapidly growing ovarian squamous cell carcinoma successfully controlled by weekly paclitaxel-carboplatin administration. Gynecol Oncol 2000;79:515-8.

14. Todo Y, Minobe S, Sasaki M, Yamamoto R, Sakuragi N. A case of puretype ovarian squamous cell carcinoma for which combination chemotherapy consisting of paclitaxel and carboplatin was not effective. Gynecol Oncol 2005;97:223-7

15. Kim HS, Kim JW, Chung HH, Park NH, Song YS, Kang SB. Disease confined within the ovary and smaller amount of ascites are good prognostic factors for survival of patients with squamous cell carcinoma arising from mature cystic teratoma of the ovary: a case series in Korea and review of the published reports. J Obstet Gynaecol Res 2009;35:99-105. 